\title{
Freeman-Sheldon syndrome: a disorder of congenital myopathic origin?
}

\author{
J VANĚK, J JANDA, V AMBLEROVÁ, AND F LOŠAN \\ From the Clinic of Orthopaedics, Department of Pathological Anatomy, Clinic of Neurology, and \\ Department of Human Genetics, Faculty Hospital, Plzeñ, Czechoslovakia.
}

SUMMARY Freeman-Sheldon syndrome was diagnosed in an unrelated adult man and woman, with severe abnormalities of the extremities but only slight anomalies of the face. Electromyography and muscle biopsy showed a myopathy which was classified as a congenital disproportion of fibre type and seemed to be the primary cause of the deformities. This allowed classification of the syndrome as a separate type of myopathic arthrogryposis.

The Freeman-Sheldon syndrome (craniocarpotarsal dysplasia or whistling face syndrome) is characterised by three basic abnormalities: microstomia with pouting lips, camptodactyly with ulnar deviation of the fingers, and talipes equinovarus. However, the expression of individual symptoms is rather variable. $^{1-3}$ Other associated abnormalities are less specific for the syndrome and their occurrence less regular, but they are diagnostically valuable. They are immobile face, superciliary ridge, deeply set eyes, strabismus, small nose, long philtrum, micrognathia, short and large neck, short stature, and scoliosis. Intelligence is usually normal.

The syndrome is relatively rare and according to Grasshoff $e t a^{1}$ only 34 patients have been reported including those in their study. Cases appear sporadically, but families with autosomal dominant ${ }^{13}$ as well as autosomal recessive ${ }^{4}$ inheritance have been described.

We report the detailed myological examination that was performed in two unrelated patients. The muscle findings could be of significance in elucidating the pathogenesis of the face and limb deformities in the Freeman-Sheldon syndrome.

\section{Case reports}

CASE 1

Case 1 was a 30 year old male. His mother had had one abortion. No congenital abnormalities were observed in any other member of the family. His height was $168 \mathrm{~cm}$ and weight $69 \mathrm{~kg}$. He had repeated surgery for bilateral talipes equinovarus and cleft palate. Microstomia was slight but became

Received for publication 16 July 1982.

Revised version accepted for publication 29 March 1985. evident on opening the mouth. The face is shown in fig 1. Radiographs of the skull were normal. The ulnar deviation of the fingers on the right hand and the substantially slighter involvement of the left hand can be seen in fig 2 . The muscles of both forearms were hypotrophic. The radius and ulna had strikingly thin diaphyses on $x$-ray. The elbows were normal apart from pronation and supination which were limited to half. The hair was abnormally fine and grew low on the back of the neck. Intelligence and the results of current laboratory examinations were within normal limits.

CASE 2

Case 2 was a 26 year old female. Her father died of heart disease at the age of 39 and her mother had

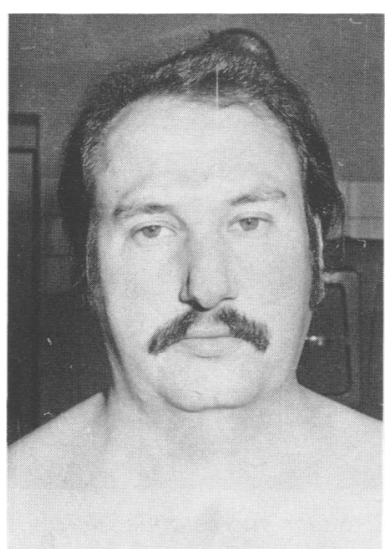

FIG 1 Case 1. Superciliary ridge and wide, short neck. Microstomia is evident only on opening the mouth. 


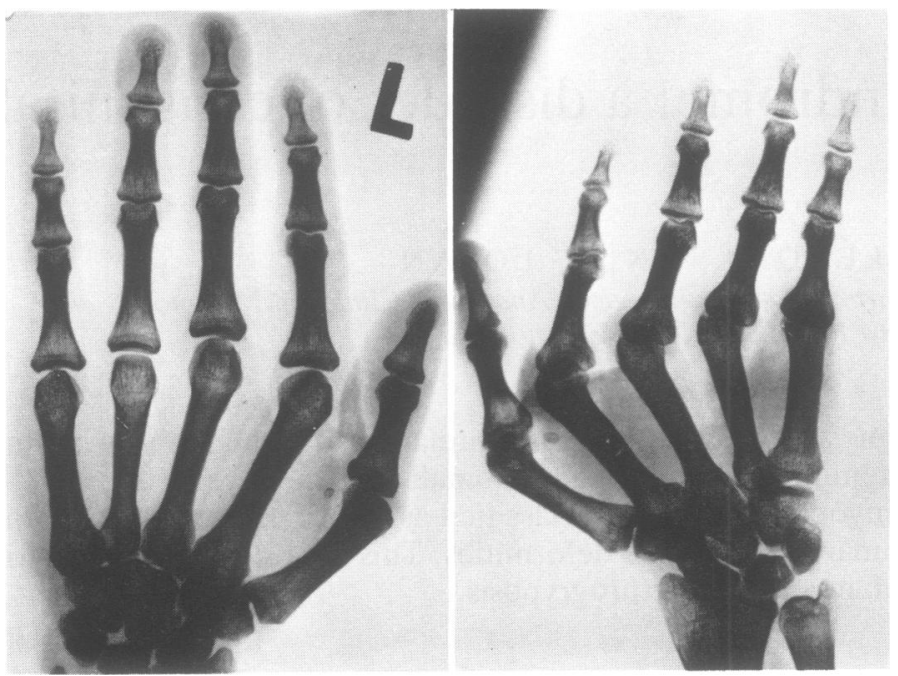

FIG 2 Case 2. Asymmetrical involvement of the hands. Pronounced bilateral divergence of metacarpals and severe ulnar deviation of fingers on the right hand only.

worked with carbide during the pregnancy. The pregnancy and delivery were uncomplicated. There were no congenital anomalies in the parents or in the brother and sister.

At the age of 23 case 2 delivered a boy who died of immaturity after 15 minutes, but no malformations were found. The next year she delivered a boy at term who died of respiratory insufficiency after 24

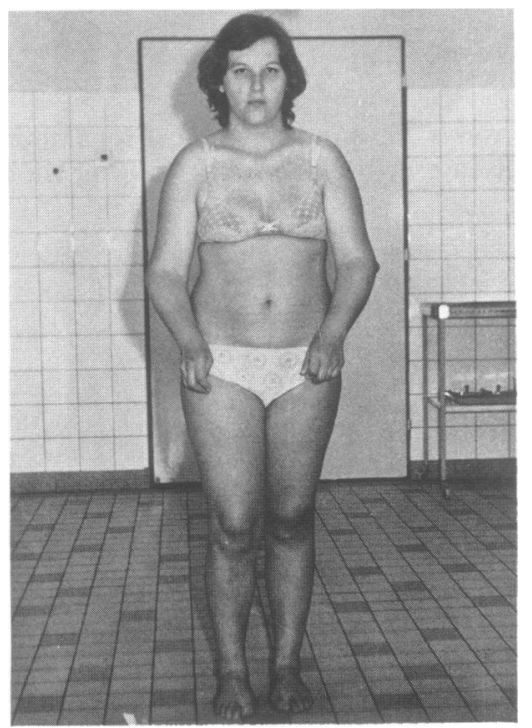

FIG 3 Case 2. Slight microstomia, deeply set eyes, short, large neck, round shoulders with low axillary folds, flexion contracture of the elbows with prominent dislocated heads of the radii, and large, smooth cubital fossae. hours. He had a flexion deformity of the third fingers of both hands.

Her height was $159 \mathrm{~cm}$ and weight $59 \mathrm{~kg}$. Fig 3 shows the face and upper limb abnormalities. Her

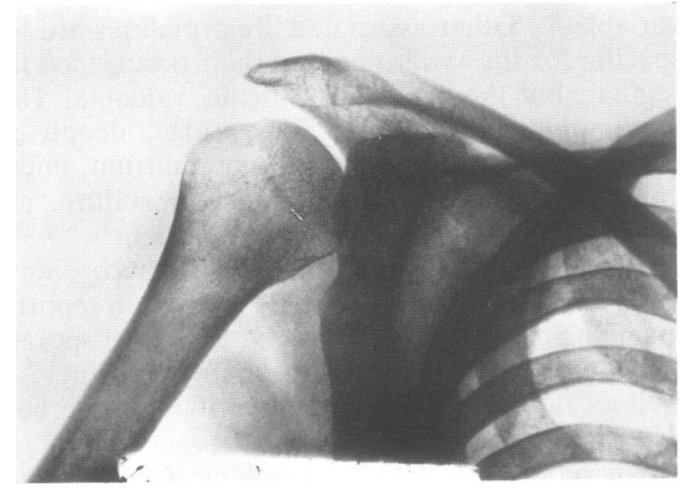

FIG 4 Case 2. Hypoplasia of the glenoid fossa.

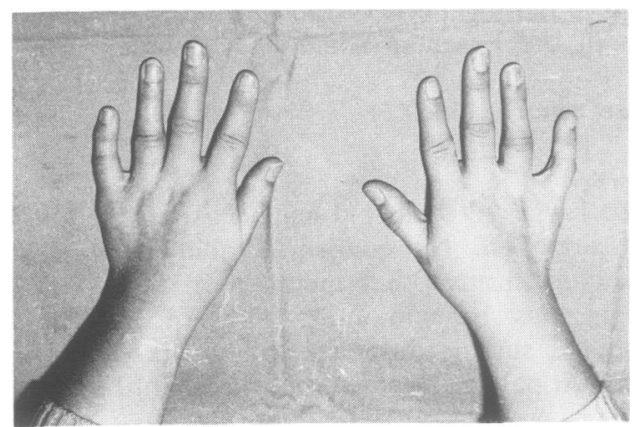

FIG 5 Case 2. Large hands with adducted thumbs. 
intelligence was normal. The mouth opening was small and she had a high arched palate. There was an abscess on the right auricle. Radiographs of the skull were normal. The glenoid fossae were hypoplastic (fig 4) but movement of the shoulder joints

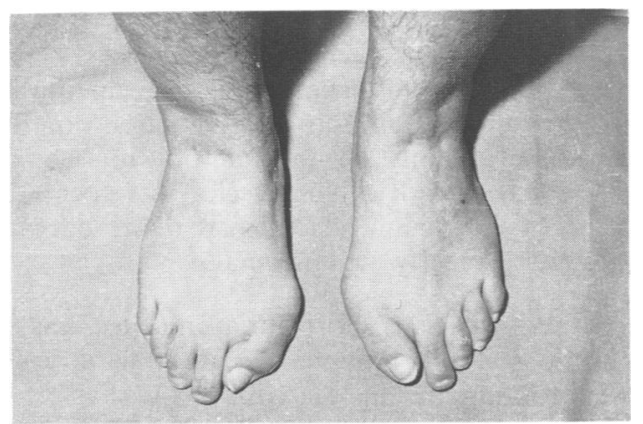

FIG 6 Broad feet and halluces valgi. was normal. Supination was not possible in the elbows and extension was limited to $45^{\circ}$. $X$-ray revealed posterior dislocation of the heads of the radii and shallow ulnar trochleae. The hands were large with adducted thumbs (fig 5). With dorsal flexion of the wrist, full extension of the interphalangeal joints of the fourth and fifth fingers was not possible. Examination of the feet showed valgus deformity of the big toes bilaterally (fig 6). With the exception of spina bifida of $\mathrm{C7}$ and coxa valga, no other abnormalities were found.

\section{DERMATOGLYPHIC STUDY}

There was an aberrant simian line on the palms of both patients. Case 1 had whorls on the first, fourth, and fifth fingertips and ulnar loops on the other fingers of both hands. Case 2 had ulnar loops on all fingertips.
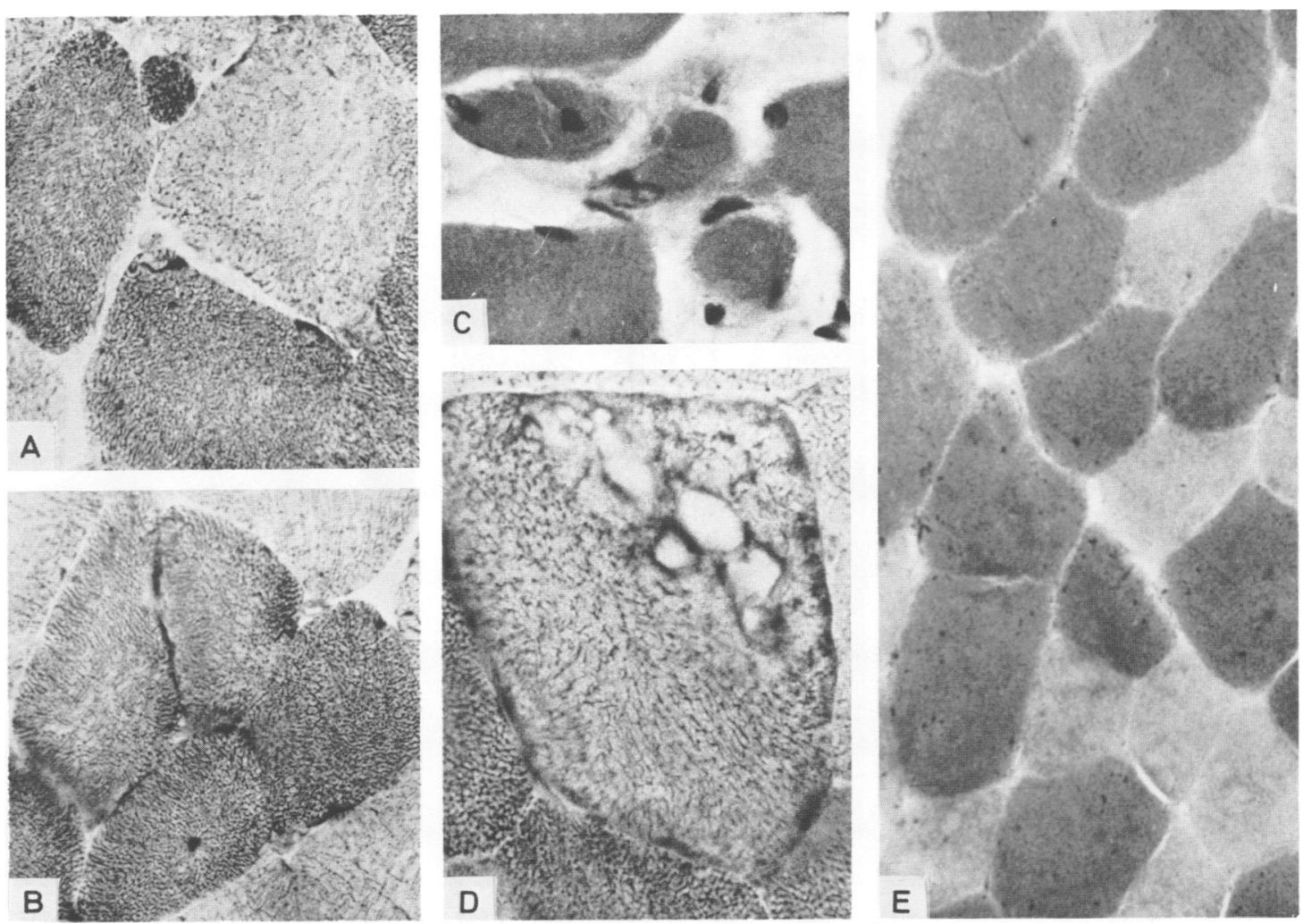

FIG 7 Case 1. Muscle biopsy. (A-D) Abnormalities of type I fibres. (A) Round atrophic fibre with high dehydrogenase activity. (NADH-TR.) (B) Internal nucleus. (NADH-TR.) (C) Round atrophic fibre with internal nucleus. (HE.) (D) Vacuolar changes. (NADH-TR.) (E) Hypertrophy of type II fibres (dark). (Routine ATPase.) 


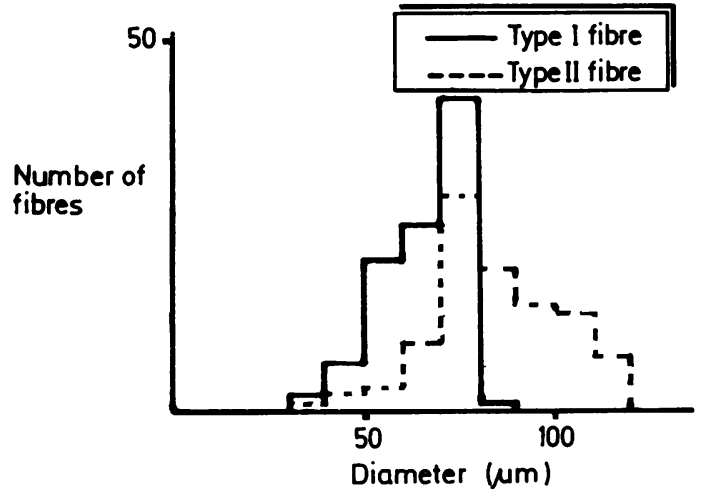

FIG 8 Case 1. Histogram of muscle fibres. Relative increase of number of type I fibres to $49.5 \%$ with mean diameter $70.6 \mu \mathrm{m}$ and hypertrophy of type II fibres with mean diameter $\overline{8} \overline{8} \mu \mathrm{m}$ (normal $65^{-\mu m}$ ). Hypertrophy index $H 2=587$.

\section{EMG FINDINGS}

Clinical neurological findings were normal in both cases. The deltoid muscles, extensor digitorum, abductor digiti minimi manus, quadriceps, and tibialis anterior were examined in both cases. Nerve conduction velocity was measured in the ulnar and peroneal nerves.

Case 1. Narrow action potentials of $3 \mathrm{~ms}$ duration and superimposed and polyphasic action potentials were found mainly in the deltoid muscle. The interference pattern of all muscles was normal and no signs of neurogenic lesions were observed. Nerve conduction velocity was normal.

Case 2. Potentials of short duration (2 to $3 \mathrm{~ms}$ ) and superimposed and polyphasic potentials appeared, most prominently in the distal muscles of the extremities. There was no sign of neurogenic lesions. Nerve conduction velocity was normal.
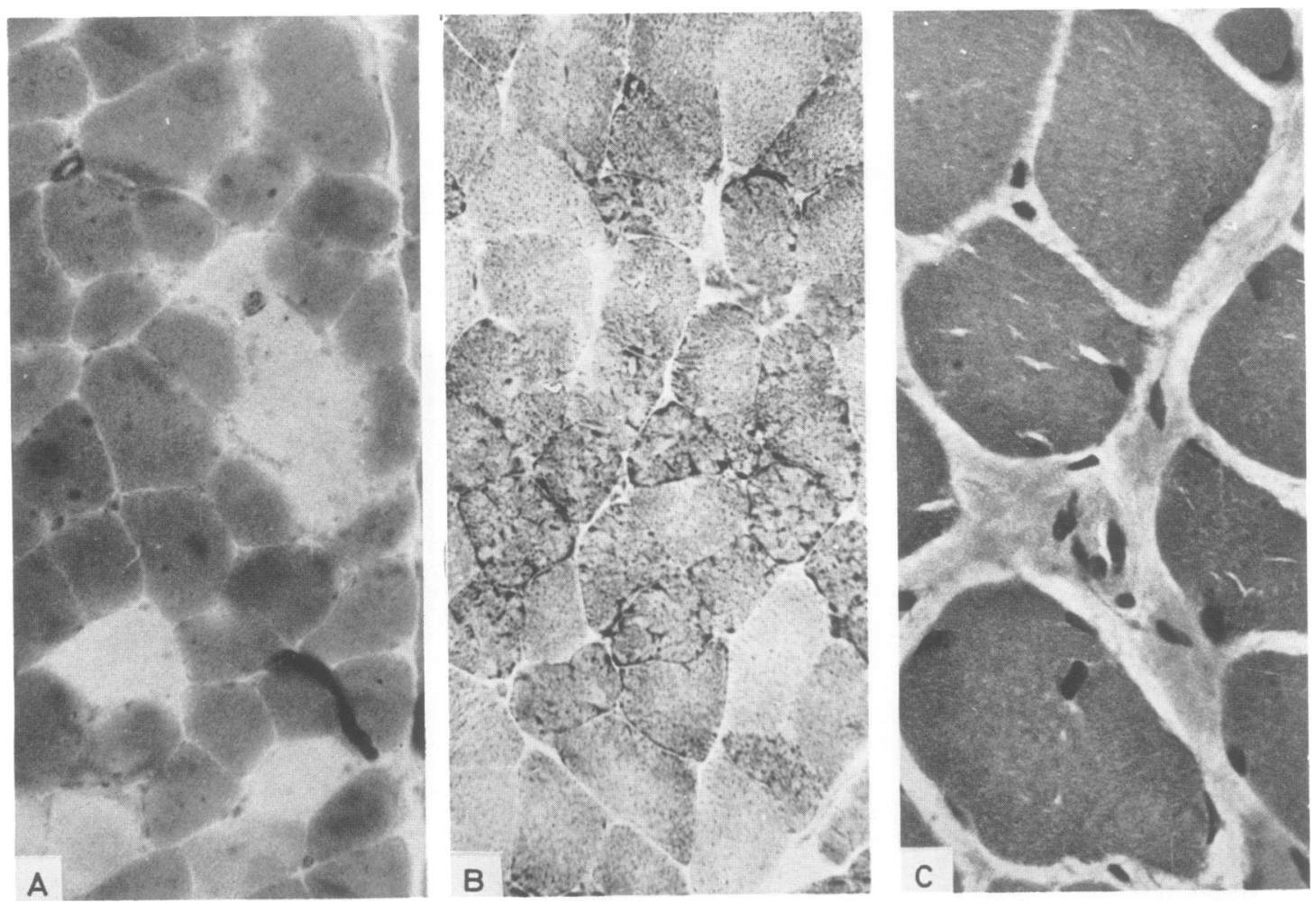

FIG 9 Case 2. Muscle biopsy. (A) Type I fibres (dark) predominate. (ATPase preincubated at pH 4.3.) (B) Irregular distribution of coarse formazan granules in type I fibres ('moth-eaten' appearance). (NADH-TR.) (C) Variation of fibre diameter with isolated, round, atrophic fibre (top right). In the narrow zone close to the myotendineous junction there is an internal nucleus. (HE.) 
These findings suggest a myopathic cause of the muscle lesions.

\section{MUSCLE BIOPSY}

Muscle biopsies were taken from deltoid muscle in case 1 and from the common origin of the extensor muscles of the forearm in case 2 . Biopsy specimens were processed for light microscopy and histochemistry. Routine ATPase sections were used for quantitative examination ${ }^{5}$ (figs 7 to 10). Electron microscopy was performed in case 1 and showed non-specfic changes including occasional central nuclei, focal $\mathrm{Z}$ line streaming and condensation, dilatation of the cisterns of the sacroplasmic reticulum, and focal enlargement of mitochondria.

The predominant involvement of type I fibres with structural changes in both cases confirms a primary myopathic origin of the observed defects. The common denominator was revealed by quantitative examination. The mean diameter of type I fibres was lower than that of type II fibres and the difference in both cases was greater than $12 \%$. This

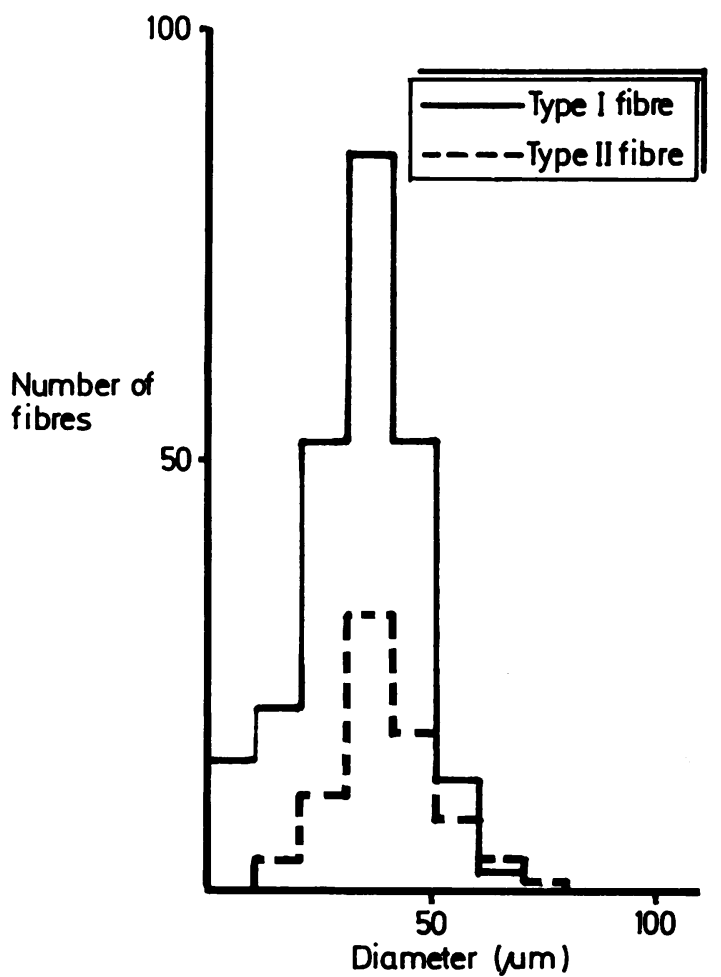

FIG 10 Case 2. Histogram of muscle fibres. Type I fibres predominate $(78 \%)$ with atrophy. Diameter $37 \mu m$ (normal $53 \mu \mathrm{m})$. Atrophy index $\mathrm{AI}=165$. Mean diameter of type $I I$ fibres $44 \mu \mathrm{m}$. result leads to the conclusion that the muscle lesion is a form of congenital fibre type disproportion.

\section{Discussion}

CLINICAL AND RADIOLOGICAL DIAGNOSIS

The three major clinical signs of the FreemanSheldon syndrome may not always be present. Rinsky and Black ${ }^{2}$ analysed 23 published cases and noted the absence of equinovarus in five patients and the absence of microstomia in one patient. However, the ulnar deviation and the flexion contractures of the fingers were present in all these patients, but even the complete absence of these in one patient has been reported recently by Wettstein et al. ${ }^{3}$ Ulnar deviation of the fingers occurs in only the most severe form of the syndrome as in our patients (figs 2 and 5). In case 2 a less marked involvement was present in the hands and thus the finding in the feet of pes planus with hallux valgus (fig 6) is not surprising.

\section{DERMATOGLYPHIC FINDINGS}

Several authors ${ }^{146}$ have mentioned non-specific dermatoglyphic changes. The dermatoglyphs of both our cases appear to correlate well with these findings.

\section{EMG FINDINGS}

Electromyographic muscle changes in the FreemanSheldon syndrome have been studied repeatedly. Sharma and Tandon ${ }^{7}$ found a decrease of activity in the buccinator muscle. MacLeod and Patriquin ${ }^{8}$ showed denervation changes in the acral muscles of the hands and feet. Žižka et al ${ }^{9}$ found noncharacteristic signs of a peripheral motor neurone lesion in the abductor brevis and orbicularis oris. Investigations by Sauk $e t a^{10}$ revealed no functioning motor units in the alar muscles of the nose. They recorded the activity of single fibres only.

Our patients showed a myogenic lesion in most of the muscles examined, with predominantly proximal involvement in case 1 and distal involvement in case 2 .

\section{MUSCLE BIOPSIES}

Biopsies processed for conventional light microscopy often fail to show early dystrophic changes. ${ }^{5}$ On the other hand, diffuse atrophy in the buccinator ${ }^{37}$ with fibrosis, ${ }^{6}$ and increase of adipose tissue and fibrosis in the gastrocnemius, ${ }^{2}$ might be late signs of muscular dystrophy.

Signs of congenital fibre type disproportion (CFTD) were clearly shown in our cases by the selective involvement of type I fibres and the mean diameter of type II fibres raised by $12 \%$. The finding 
of CFTD excludes the secondary muscle changes of neurogenic origin or disuse. CFTD belongs to a group of congenital myopathies the diagnosis of which is based on quantitative muscle examination and is often associated with abnormalities of joints and stature. ${ }^{511}$ The myopathic aetiology of the Freeman-Sheldon syndrome has previously been suggested by Sauk et l $^{10}$ and their suggestion, based only on findings in the facial muscles, appears now to be confirmed.

Sauk et $a l^{10}$ quote a personal communication of Fraser, who observed an alteration of the pulmonary volume in the syndrome and suggested an intercostal myopathy. This could be connected with bilateral bronchopneumonia, which was observed in a child after surgery. ${ }^{8}$ We found reports of severe respiratory infection in four patients, of whom two died. Wettstein $e t a l^{3}$ also referred to a child who died of bronchopneumonia. The second child of our case 2 died of respiratory insufficiency shortly after delivery. It may be that the respiratory problems and the abnormal flexed position of the third digits represent the only evident manifestation of the mild form of the syndrome.

The danger of respiratory complications and the high resistance of talipes equinovarus to conservative and even operative treatment ${ }^{2}$ are two significant therapeutic problems that must be taken into consideration when dealing with the syndrome.

From correlation of clinical signs, EMG, and muscle biopsy findings it seems that FreemanSheldon syndrome is caused by a non-progressive or slowly progressive myopathy characterised by con- genital fibre type disproportion with predominantly facial, limb, and possibly respiratory muscle involvement. In our opinion, Freeman-Sheldon syndrome represents an independent type of arthrogryposis multiplex congenita.

\section{References}

1 Grasshoff H, Braun HSt, Sakreida M. Beitrag zum FreemanSheldon Syndrome. Beitr Orthop Traumatol 1978;25:241-7.

2 Rinsky LA, Black EE. Freeman-Sheldon ('whistling face') syndrome. J Bone Joint Surg [Am/ 1976;58:148-50.

3 Wettstein A, Buchinger G, Braun A, Bazan UB. A family with whistling-face syndrome. Hum Genet 1980;55:177-89.

4 Kousseff BG, McConnachie P, Hadro TA. Autosomal recessive type of whistling face syndrome in twins. Pediatrics 1982;69: 328-31.

5 Dubowitz V, Brooke MH. Muscle biopsy. A modern approach. Philadelphia: Saunders, 1973.

6 Weinstein S, Gorlin RJ. Cranio-carpo-tarsal dysplasia or the whistling-face syndrome. I. Clinical considerations. Am J Dis Child 1969;117:427-33

7 Sharma RN, Tandon SN. 'Whistling face' deformity in craniofacio-corporal syndrome. $\mathrm{Br}$ Med J 1970;4:33.

${ }^{8}$ MacLeod P, Patriquin H. Whistling face syndrome-craniocarpo-tarsal dysplasia. Clin Pediatr 1974;13:184-9.

${ }^{9}$ Žižka J, Lichý J, Balíček P. Syndrome of cranio-carpo-tarsal dysplasia in a 6-year-old girl (whistling-face syndrome). Cesk Pediatr 1979;34:96-7.

11 Sauk JJ, Delaney JR, Reaume C, Brandjord R, Witkop CJ. Electromyography of oral-facial musculature in cranio-carpal dysplasia (Freeman-Sheldon syndrome). Clin Genet 1974;6: 132-7.

$"$ Swash M, Schwartz MS. Neuromuscular diseases. A practical approach to diagnosis and management. Berlin: SpringerVerlag, 1981:189-90.

Correspondence and requests for reprints to $\operatorname{Dr} \mathrm{J}$ Vaněk, Čechova 51, 32028 Plzeň, Czechoslovakia. 